\title{
High-performance photoacoustic probe for biopsy-free assessment of copper status in murine models of Wilson's disease and liver metastasis
}

Melissa Y. Lucero, Yuqi Tang, Chloe J. Zhang, Shengzhang Su, Joseph A. Forzano, Valeria Garcia, Xin Huang, David Moreno, Jefferson Chan*

Department of Chemistry, The Beckman Institute for Advanced Science and Technology, University of Illinois at Urbana-Champaign, Urbana, IL, USA

Jefferson Chan

Email: jeffchan@illinois.edu

Author Contributions: M.Y.L. synthesized PACu-1. Y.T., C.J.Z., V.G., X.H., and D.M. helped to synthesize PACu-1 analogs. J.A.F. synthesized RPS1. M.Y.L. performed all in vitro characterization experiments. M.Y.L. performed all animal imaging with help from S.S. and C.J.Z. M.Y.L. and S.S. conducted the blind BFA study. M.Y.L. and J.C. analyzed the data and prepared the manuscript.

Competing Interest Statement: The authors have no competing interests.

Classification: Chemistry

Keywords: Photoacoustic imaging, copper, Wilson's disease

This PDF file includes:

Main Text

Figures 1 to 6 


\begin{abstract}
The development of high-performance photoacoustic (PA) probes that can monitor disease biomarkers in deep-tissue has the potential to replace invasive medical procedures such as a biopsy. However, such probes must be highly optimized for in vivo performance and exhibit an exceptional safety profile. In this study, we have developed PACu-1, the first PA probe designed for biopsy-free assessment (BFA) of hepatic $\mathrm{Cu}$ via photoacoustic imaging. PACu-1 features a $\mathrm{Cu}(\mathrm{I})$-responsive trigger appended to an aza-BODIPY dye platform that has been optimized for ratiometric sensing. Owing to its excellent performance, we were able to detect basal levels of $\mathrm{Cu}$ in healthy wildtype mice, as well as elevated $\mathrm{Cu}$ in a Wilson's disease model and in a liver metastasis model. To showcase the potential impact of PACu-1 for BFA, we conducted a blind study where we were able to successfully identify a Wilson's disease animal from a group of healthy control mice with greater than $99.7 \%$ confidence.
\end{abstract}

\title{
Significance Statement
}

The ability to non-invasively detect and track disease biomarkers via photoacoustic imaging can potentially serve as a substitute for invasive medical procedures such as a liver biopsy. While achieving this goal can have a profound impact on disease management, it is an immense challenge that requires novel chemical tools that are sensitive, selective, and safe. Here we report an acoustogenic probe designed for $\mathrm{Cu}(\mathrm{I})$, which becomes dysregulated in many disease states. In addition to demonstrating in vivo efficacy in multiple models, we designed a blind study to assess its utility for biopsy-free assessment of hepatic copper levels in Wilson's disease. This work sets the stage for future studies to evaluate the performance of acoustogenic probe designs for biomedical applications.

\section{Main Text}

\section{Introduction}

Photoacoustic (PA) imaging is a light-in, sound-out technique that has emerged as a promising biomedical approach for the non-invasive assessment of various ailments in humans, ranging from arthritis to cancer. ${ }^{1,2}$ Excitation of an endogenous pigment such as hemoglobin in blood or melanin in tissue can provide contrast since relaxation via non-radiative decay can trigger thermoelastic expansion of the surrounding tissue. Repeatedly irradiating a region of interest with a pulsed laser can result in pressure waves that can be readily detected by ultrasound transducers. Since ultrasound at clinically relevant frequencies can travel through the body with minimal perturbation, it is possible to accurately pinpoint the source of the signal to afford high resolution images at centimeter imaging depths. ${ }^{3}$ Beyond label-free applications, the utility of PA imaging for disease detection has been augmented by the recent development of acoustogenic probes (activatable PA probes) that give an off-on signal enhancement or ratiometric readout. ${ }^{4,5}$ Notable examples include those that can visualize dysregulated enzymatic activities ${ }^{6-9}$ properties of the disease tissue microenvironment, ${ }^{10-12}$ as well as small molecule- and metal ion-based disease biomarkers. ${ }^{13-18}$ However, replacing an invasive medical procedure such as a liver biopsy with an acoustogenic probe is an immense challenge since the in vivo performance and safety profile of such a chemical tool must be exceptional. Thus, in spite of undesirable shortcomings such as the potential to develop severe infections, false negatives due to collection of nondiseased tissue, and the inability to directly monitor disease progression in real-time, ${ }^{19}$ liver biopsies are still commonly employed to assess biomarkers in conditions such as Wilson's disease (WD) ${ }^{20}$ and cancer. ${ }^{21}$ 
It is noteworthy that elevated levels of hepatic copper $(\mathrm{Cu})$ is a common biomarker shared by these conditions. In WD, Cu accumulates in the liver due to a genetic mutation in the Cu-exporter, ATP7B, and this can lead to chronic liver damage which can become fatal if not treated. ${ }^{22,}{ }^{23}$ In the context of cancer, $\mathrm{Cu}$ is elevated in many solid tumors including breast ${ }^{24,25}$ and lung ${ }^{26,27}$ cancers which generally metastasize to the liver. Since $\mathrm{Cu}$ can promote angiogenesis and drive tumor progression, BFA of $\mathrm{Cu}$ in metastatic lesions is critical. While several probes have been developed for in vivo imaging of $\mathrm{Cu}$, these early examples were designed for fluorescent ${ }^{28}$ and bioluminescent ${ }^{29}$ methods which are more suitable for shallow imaging depths ( $\mathrm{mm}$ range) owing to scattering and attenuation of light. More recently, our group ${ }^{13,30}$ and others ${ }^{31}$ have developed $\mathrm{Cu}$ probes for PA imaging to achieve greater tissue penetration and higher resolution. However, these probes are designed to target $\mathrm{Cu}(\mathrm{II})$, whereas intracellular $\mathrm{Cu}$ exists predominantly in the +1 -form owing to a highly reducing environment of the cell. ${ }^{32}$ To overcome this challenge, we present the development of PACu-1, the first acoustogenic probe for $\mathrm{Cu}(\mathrm{I})$ and its application in BFA of hepatic $\mathrm{Cu}$ in a WD model and a liver metastasis model. Moreover, we designed an unbiased BFA blind study to identify a Wilson's disease mouse from a group of healthy wildtype controls using PACu-1.

\section{Results}

\section{Design and Characterization of PACu-1}

To target the +1-oxidation state of $\mathrm{Cu}$, we installed a $\mathrm{Cu}(\mathrm{I})$-responsive tris[(2-pyridyl)methyl]amine $(\mathrm{TPA})^{33}$ trigger onto an optimized aza-BODIPY dye platform to yield PACu-1 which features ratiometric imaging capabilities. Specifically, we hypothesized capping of the 2,6-dichlorophenol moiety will result in a blue-shift of the wavelength of maximum absorbance $\left(\lambda_{\max }\right)$ relative to the uncapped probe. However, the binding of $\mathrm{Cu}(\mathrm{I})$ to TPA will induce an oxidative cleavage event of the pendant ether linkage to release the latent dye (Figure 1a). Subsequently, selective irradiation of each form (probe and product) at their corresponding $\lambda_{\max }$ will yield two signals, from which a ratio can be determined. This probe design feature is important for BFA, especially in the liver since we anticipate there to be significant background interference from blood. In addition, we selected the aza-BODIPY platform to develop PACu-1 due to their large extinction coefficients $\left(10^{4}\right.$ to $\left.10^{5} \mathrm{M}^{-1} \mathrm{~cm}^{-1}\right)$ in the near infrared range and low fluorescence quantum yields since both of properties translate to a stronger PA signal. ${ }^{30}$ Lastly, we have determined empirically that many of the aza-BODIPY-based probes we have developed intrinsically localizes to the liver owing to its relatively high hydrophobic properties.

After synthesizing PACu-1 (Scheme S1), we evaluated its in vitro response to 20 equivalents of $\mathrm{Cu}(\mathrm{I})$ (introduced as $\left[\mathrm{Cu}\left(\mathrm{CH}_{3} \mathrm{CN}\right)_{4}\right] \mathrm{PF}_{6}$ ). After $1 \mathrm{~h}$ incubation at room temperature, we observed a large spectral shift of $91 \mathrm{~nm}$ from $678 \mathrm{~nm}$ (probe, $\varepsilon=5.3 \times 10^{4} \mathrm{M}^{-1} \mathrm{~cm}^{-1}$ ) to $767 \mathrm{~nm}$ (product, $\varepsilon=$ $3.7 \times 10^{4} \mathrm{M}^{-1} \mathrm{~cm}^{-1}$ ) (Figure 1b). Given that the extinction coefficient of a molecule is a reliable proxy for its PA output, we estimate the ratiometric turn-on will be $\sim 10.4$-fold (defined as $\left.\left(770 / 680_{\text {Final }}\right) /\left(770 / 680_{\text {Initial }}\right)\right)$. Importantly, irradiation at $680 \mathrm{~nm}$ will predominately excite PACu-1, whereas light at $770 \mathrm{~nm}$ will only generate a signal that corresponds to the turned over product (Figure 1c-d). Moreover, we were able to observe a dose-dependent response to Cu (LOD $=0.2$ $\mu \mathrm{M}$ ) (Figure 1e). We were also able to show that PACu-1 can function in the presence of glutathione (GSH), an abundant biological thiol, that can compete with PACu-1 to bind $\mathrm{Cu}(\mathrm{I})$ (Figure 1f). Indeed, GSH is present at high levels in the liver and most solid tumors (up to 10 $\mathrm{mM}){ }^{34,}{ }^{35}$ Lastly, PACu-1 was shown to exhibit excellent selectivity for $\mathrm{Cu}(\mathrm{I})$ against a panel of monovalent and divalent metal ions (Figure 1g). This finding is significant because in addition to $\mathrm{Cu}(\mathrm{I})$, the TPA trigger has been tuned to sense other metal ions and thus, may exhibit off-target reactivity. ${ }^{36,37}$

\section{Metabolic Stability, Biodistribution, and Safety Profile of PACu-1}


Because our objective is to employ PACu-1 for BFA of hepatic Cu via PA imaging, it is critical to 1) demonstrate that it is not metabolized in the liver to give false positives and 2) show that it is biocompatible with an excellent safety profile. To this end, we treated PACu-1 with rat liver microsomes rich in metabolic enzymes (e.g., CYP450s). After an incubation period of $1 \mathrm{~h}$, we did not observe any change in the absorbance spectra indicating there would be minimal off-target activation of PACu-1 that can lead to false positive results (Figure S1). We corroborated these results with mass spectroscopy analysis that showed the latent aza-BODIPY was not being released. Next, we performed MTT assays to assess the cytotoxicity of the probe in mammalian cell lines. For instance, HEK293 cells incubated with up to $25 \mu \mathrm{M}$ of PACu-1 for $24 \mathrm{~h}$ were shown to have no significant loss of viability (Figure 2a). Next, we sought to determine the biodistribution of PACu-1 after systemic administration in BALB/c mice via ex vivo PA imaging analysis of the vital organs. Our data indicates that PACu-1 predominantly localizes to the liver and does not accumulate in the heart, kidneys, or spleen (Figure S2). Before PACu-1 could be considered further as a chemical tool for BFA applications, we examined its in vivo safety profile. First, we performed $\mathrm{H} \& \mathrm{E}$ staining on liver samples obtained from mice treated with either a vehicle control or PACu-1. Our results show that the nuclear staining patterns were identical, suggesting that PACu-1 is non-toxic (Figure 2b). Second, we conducted a comprehensive liver function test to measure the levels of albumin, alkaline phosphatase (ALP), alanine aminotransferase (ALT), aspartate transaminase (AST), bilirubin, blood urea nitrogen (BUN), cholesterol, and glucose in serum. We did not observe any statistical difference between vehicle- and PACu-1-treated animals which further demonstrates that PACu-1 is safe and thus, is ideal for BFA applications (Figure 2c).

\section{Imaging Exogenous $\mathrm{Cu}(\mathrm{I})$ in $\mathrm{BALB} / \mathrm{c}$ Mice}

To determine whether PACu-1 can be employed to detect elevated hepatic $\mathrm{Cu}(\mathrm{I})$ in live animals, we treated $B A L B / c$ mice with $\mathrm{CuCl}_{2}$ via intraperitoneal administration $2 \mathrm{~h}$ prior to the introduction of PACu-1. Of note, $\mathrm{Cu}(\mathrm{II})$ is rapidly reduced to $\mathrm{Cu}(\mathrm{I})$ upon uptake into cells. PA imaging revealed that the $\mathrm{PA}_{770 / 680}$ ratio was $1.48 \pm 0.23$ for the Cu-treated animals, whereas the corresponding ratio for the vehicle control was $0.94 \pm 0.13$ (Figure 3, red and blue, respectively). To confirm that these results were due to the detection of $\mathrm{Cu}(\mathrm{I})$, we administered ammonium tetrathiomolybdate (TM), a high affinity FDA-approved Cu chelator drug $\left(K_{\mathrm{d}}=\sim 10^{-20}\right),{ }^{38}$ prior to treatment with PACu1. As anticipated, we did not observe any activation $(0.94 \pm 0.13)$ when TM was present since it can outcompete PACu-1 for binding to $\mathrm{Cu}(\mathrm{I})$ (Figure 3, yellow). To further validate this finding, we administered Ctrl-PACu-1, a non-responsive control probe that features an attenuated $\mathrm{Cu}(\mathrm{I})$ binding trigger (Scheme S2), to a fourth group of animals. Interestingly, PA imaging demonstrated that the ratio was also lower than both the vehicle group and the TM group $(0.82 \pm$ 0.12) (Figure 3, green). The lower ratio suggests that PACu-1 can detect basal levels of $\mathrm{Cu}$ that are present in the liver. Finally, we performed ICP-MS analysis on liver samples obtained from mice treated with $\mathrm{CuCl}_{2}$. Compared to animals that received a vehicle control, the concentration of hepatic Cu was twice as high (Figure 3c).

\section{PA Imaging of Hepatic $\mathrm{Cu}(\mathrm{I})$ in Wilson's Disease}

$\mathrm{Cu}$ accumulation in the liver is a pathological hallmark of WD which is typically assessed clinically via liver biopsies. ${ }^{39}$ Using an established ATP7B genetic knockout model of WD developed by Lutsenko and co-workers (JAX stock \#032624), we measured the levels of hepatic $\mathrm{Cu}$ in wildtype mice and WD mice using ICP-MS analysis after obtaining biopsied tissue. On average, we found that the $\mathrm{Cu}$ levels in WD mice were 17.5-fold greater than wildtype mice (Figure 4a). Likewise, when we employed PACu-1 and PA imaging for BFA of $\mathrm{Cu}$, we found that the PA ${ }_{770 / 680}$ ratio was significantly higher in WD mice $(1.24 \pm 0.16)$ relative to wildtype mice $(0.80 \pm 0.11)$ (Figure $4 \mathbf{b}-\mathbf{d})$. It is critical to note that while ICP-MS analysis reports on total Cu levels, PACu-1 can only access the labile pool which is defined as Cu weakly associated with intracellular chelators such as GSH. To confirm the in vivo imaging results, we harvested the heart, kidneys, liver, and spleen from WD and wildtype mice treated with PACu-1 to perform ex vivo PA imaging. This experiment was performed to demonstrate that the PA signal intensity is higher in the liver of WD mice owing to activation of PACu-1 (Figure S3). 


\section{BFA of Hepatic $\mathrm{Cu}(\mathrm{l})$ in Wilson's Disease via a Blind Study}

To evaluate the potential efficacy of PACu-1 for BFA of hepatic $\mathrm{Cu}(\mathrm{I})$ in $\mathrm{WD}$, it is critical to perform a rigorous study that is free of potential bias. To this end, we designed a blind experiment where one investigator randomly selected mice belonging to either the WD or wildtype groups (eight total) for the study (Figure 5a). Each of the animals were then tagged, and their identities were concealed until the completion of the study. A second investigator then administered PACu1 and employed PA imaging to identify the WD mice. There was no physical indicator that would allow us to distinguish the mice based on appearance. Prior to BFA, a reliable diagnostic threshold was determined in wildtype mice, which is defined as the $\mathrm{PA}_{7706680}$ ratio $(0.82 \pm 0.10)$ (vide infra). With this in mind, we identified seven animals with a $\mathrm{PA}_{770 / 680}$ ratio $(0.63,0.72,0.82$, $0.86,0.87,0.93,0.94)$ within two standard deviations of the diagnostic threshold which were assigned to Group 1 (wildtype mice) (Figure 5b-d). In contrast, only one of the animals had a $\mathrm{PA}_{770 / 680}$ ratio (1.16) greater than three standard deviations of the diagnostic threshold and was correspondingly assigned to Group 2 (WD mouse). When the identity of the eight animals were revealed at the end of the study, we were able to correctly identify the WD mouse with greater than $99.7 \%$ confidence.

\section{PA Imaging of $\mathrm{Cu}(\mathrm{I})$ in a Liver Metastasis Model}

Finally, we turned our attention to a second model to further showcase the potential clinical impact of PACu-1. Elevated $\mathrm{Cu}$ in cancer of the bone, breast, gastrointestinal tract, and lungs has been associated with aggressive phenotypes and poorer prognosis. ${ }^{25}$ There are ongoing efforts to employ $\mathrm{Cu}$ chelation therapy to reduce the copper status in primary tumors, as well as in metastatic lesions to treat cancer ${ }^{40,}{ }^{41}$ Since the liver is one of the most common sites of metastasis in the body, BFA of $\mathrm{Cu}(\mathrm{I})$ levels would facilitate real-time monitoring during tumor progression and treatment with a chelator. Nu/J mice were either implanted with A549 cells in the liver or received sham surgeries. After four weeks, PACu-1 was administered for PA imaging. We elected to use a PA instrument (MSOT inVision, iThera Medical) capable of whole-body crosssectional imaging for this study because a built-in feature would allow us to readily perform spectral unmixing to distinguish the signal from PACu-1 and blood. Compared to the animals that received sham surgeries $(1.06 \pm 0.28)$, the $P A$ fold turn-on (defined as $\left.P A_{\text {Final }} / \mathrm{PA}_{\text {lnital }}\right)$ of tumorbearing mice was $2.31 \pm 0.78$ (Figure 6a-c). This indicates that in addition to being able to sense hepatic $\mathrm{Cu}(\mathrm{I})$ in WD, PACu-1 can also detect elevated $\mathrm{Cu}$ in a lung cancer liver metastases model.

\section{Discussion}

One of the major goals of molecular imaging research is to develop high-performance chemical tools that can non-invasively detect and monitor disease biomarkers in a deep-tissue context. PA imaging is ideal for this application because it involves the conversion of safe near infrared light to non-toxic ultrasound waves. Since sound at clinically relevant frequencies can readily pass through the body, it is possible to obtain high resolution images beyond $10 \mathrm{~cm}$ in depth. ${ }^{42}$ Despite the emergence of various acoustogenic probes for analyte sensing, none have been explored to date for BFA of disease biomarkers of the liver. Thus, our goal is to develop PA probes that can potentially replace or complement invasive biopsies currently in use to provide real-time monitoring capabilities.

In this study, we chose to target $\mathrm{Cu}$ because while it is an essential metal ion required by all living organisms, aberrant levels are linked to genetic disorders such as WD, as well as most solid cancer types. Our group has previously developed several PA probes for $\mathrm{Cu}(\mathrm{II}),{ }^{13}, 30$ however we found that they were not stable when incubated with RLMs. Likewise, after synthesizing RPS1, a PA probe designed to image $\mathrm{Cu}(\mathrm{II})$ in a murine Alzheimer's disease model, ${ }^{31}$ we discovered that it could not detect exogenous copper in the liver (Figure S4). These results are not surprising since each of these examples were designed to respond to $\mathrm{Cu}$ in its +2-oxidation state. PACu-1 on the other hand, is highly selective for $\mathrm{Cu}(\mathrm{I})$, affords a robust PA signal enhancement when irradiated 
at $770 \mathrm{~nm}$, is compatible with ratiometric sensing, intrinsically targets the liver, and most importantly, exhibits an exceptional safety profile.

It is worth noting that one of the major differences between BFA using PACu-1 and traditional biopsies is that our probe is designed to detect the labile $\mathrm{Cu}$ pool ( $\mathrm{Cu}$ associated with $\mathrm{GSH}$ ), whereas the latter technique reports on the total $\mathrm{Cu}$ content in the sample. Despite this difference, we can still reliably distinguish WD mice from wildtype controls as shown in our blind study. In addition to detecting $\mathrm{Cu}$ in WD, we also demonstrate PACu-1 can be used to detect elevated $\mathrm{Cu}$ in a liver metastasis model. We envision PACu-1 can be used to aid in the development of new $\mathrm{Cu}$ chelators or in conjunction with existing $\mathrm{Cu}$ binding drugs to monitor changes in real-time. As previously mentioned, we employed two different PA instruments for the WD and cancer studies. This indicates that PACu-1 will be compatible with a range of imaging systems including new hand-held scanners, ${ }^{43}, 44$ wearable devices, ${ }^{45}, 46$ and endoscopic setups. ${ }^{47,}$

${ }^{48}$ Lastly, we envision this work will inspire the development of other PA probes for BFA applications.

\section{Materials and Methods}

In vitro selectivity assay. The initial absorbance $(400-800 \mathrm{~nm})$ of PACu-1 $(5 \mu \mathrm{M}, 1: 1$ DMF:HEPES, pH 7.4) was measured before the addition of a panel of metal ions $(100 \mu \mathrm{M})$. These initial measurements were used to determine the initial ratio ${ }_{770 / 680}$ via UV-vis spectroscopy. After addition, the cuvette was sealed and incubated for $1 \mathrm{~h}$. Final measurements were recorded, and the ratiometric fold turn-on was calculated by dividing the final ratio with the initial ratio. All metal solutions were prepared in water from their chloride salt, except for $\mathrm{Ag}_{2} \mathrm{CO}_{3} \cdot \mathrm{Cs}_{2} \mathrm{CO}_{3}$, and tetrakis(acetonitrile)copper(I) hexafluorophosphate.

Biopsy assessment of hepatic Cu via ICP-MS. BALB/C mice were anesthetized using isoflurane $(1.5-2.0 \%)$. The mice were then intraperitoneally injected with a solution of $\mathrm{CuCl}_{2}(5$ $\mathrm{mg} / \mathrm{kg}$ ) or vehicle (sterilized saline). After 2 hours, the mice were euthanized, then the liver was excised and weighed for ICP-MS analysis. The 2-hour incubation time was used to reduce $\mathrm{Cu}$ in vivo. To determine the $\mathrm{Cu}$ concentration in WT (B6129SF2/J) and WD (B6;129S1$\left.A t p 7 b^{t m 1 T c g} / L t s n k J\right)$ mice, the livers were similarly prepared as the BALB/c mice for ICP-MS analysis, except no intraperitoneal injections were performed.

Ex vivo biodistribution of PACu-1 via PA imaging. BALB/c mice were anesthetized using isoflurane $(1.5-2.0 \%)$ and retro-orbitally injected with either a solution of PACu-1 $(50 \mu \mathrm{M})$ or vehicle $(10 \%$ DMSO in sterilized saline, $50 \mu \mathrm{L}))$. After 1 hour, the mice were euthanized, and the liver, spleen, heart, and kidneys were excised. Photoacoustic imaging of the organs was performed at 680 and $770 \mathrm{~nm}$ using continuous mode with a 6 second rotation time (Nexus 128+, Endra Life Sciences) The ratio of the PA signals in PACu-1 treated mice obtained upon excitation at $680 \mathrm{~nm}$ and $770 \mathrm{~nm}$ were normalized to the ratio of the PA signals in vehicle treated mice.

Determination of the diagnostic threshold. A group of 10 wildtype mice (B6129SF2/J), which are direct controls of the WD mice (B6;129S1-Atp7 $\left.b^{t m 1 T c g} / L t s n k J\right)$, were used to determine the diagnostic threshold for hepatic $\mathrm{Cu}$ in Wilson's disease via PA imaging. After the mice were anesthetized using isoflurane $(1.5-2.0 \%)$, their abdomens were shaved, and they were positioned in the PA tomographer to facilitate direct imaging of the abdomen. After an image was acquired, an ROI was drawn around the liver to determine the signal intensity. The ratio of the PA signals obtained upon excitation at $680 \mathrm{~nm}$ and $770 \mathrm{~nm}$ in the ROI provided the initial PA ${ }_{770 / 680}$ ratio. The mice were then treated with a $50 \mu \mathrm{M}$ solution of PACu-1 in saline containing $10 \%$ DMSO $(50 \mu \mathrm{L})$ via retro-orbital injection. The mice were returned to their cages for 60 minutes while PACu-1 was allowed to react with the hepatic $\mathrm{Cu}$. The mice were anesthetized and their livers were imaged as described previously to obtain the final $\mathrm{PA}_{770 / 680}$ ratio. The diagnostic 
threshold value (mean $\pm 2 \times \mathrm{SD}$ ) was determined by dividing the final $\mathrm{PA}_{770 / 680}$ ratio with the initial $\mathrm{PA}_{770 / 680}$ ratio.

Identification of WD via PA imaging in a blind study. A group of eight mice consisting of one

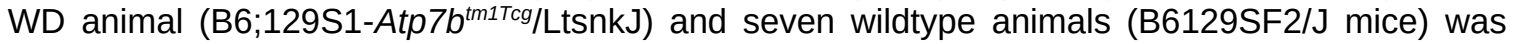
tagged and randomized by the first researcher. Their identity and the total number of WD mice present was concealed until the end of the study. Importantly, these mice had no distinguishing physical features that would allow us to identify them based on appearance. PA imaging of hepatic $\mathrm{Cu}$ using PACu-1 was then performed by a second researcher to determine the PA ratiometric fold turn-on for each animal. Mice with a $\mathrm{PA}_{770 / 680}$ ratio value greater than 1.02 was assigned to Group 1 (WD) and mice with a $P A_{770 / 680}$ ratio value between 0.62 to 1.02 was assigned to Group 2 (wildtype). After PA imaging was performed on all animals, the assignment and identity were revealed to and validated by the corresponding author.

Statistical analyses. Statistical analyses were performed in Microsoft Excel. Sample sizes in all experiments were sufficiently powered to detect at least a $p$ value $<0.05$, which was significant. All data are expressed as mean \pm SD. Multiple group analysis was performed using the KruskalWallis Test. All other in vivo imaging data was analyzed by performing the Student's t-test $(\alpha=$ 0.05). ${ }^{\star} p>0.05 ;{ }^{* \star} p>0.01$.

\section{Acknowledgments}

General: M.Y.L. thanks the Alfred P. Sloan Foundation for financial support. Major funding for the $500 \mathrm{MHz}$ Bruker CryoProbe was provided by the Roy J. Carver Charitable Trust (Muscatine, lowa; Grant No. 15-4521) to the School of Chemical Sciences NMR Lab. The Q-Tof Ultima mass spectrometer was purchased in part with a grant from the National Science Foundation, Division of Biological Infrastructure (DBI-0100085). We also acknowledge Dr. Iwona Dobrucka and the Molecular Imaging Laboratory at the Beckman Institute for use of the IVIS imaging system, the veterinary histology and diagnostic laboratories for performing H\&E staining and liver function tests, Drs. Nicole Herndon and Jessica Xu for help generating the liver metastasis model, and Prof. Svetlana Lutsenko for helpful discussions.

Funding: This work was supported in part by the National Science Foundation (1752879).

\section{References}

[1] Steinberg, I., Huland, D. M., Vermesh, O., Frostig, H. E., Tummers, W. S., and Gambhir, S. S. (2019) Photoacoustic clinical imaging, Photoacoustics 14, 77-98.

[2] Attia, A. B. E., Balasundaram, G., Moothanchery, M., Dinish, U. S., Bi, R., Ntziachristos, V., and Olivo, M. (2019) A review of clinical photoacoustic imaging: Current and future trends, Photoacoustics 16, 100144-100144.

[3] Wang, L. V., and Hu, S. (2012) Photoacoustic Tomography: In Vivo Imaging from Organelles to Organs, Science 335, 1458.

[4] East, A. K., Lucero, M. Y., and Chan, J. (2021) New directions of activity-based sensing for in vivo NIR imaging, Chemical Science 12, 3393-3405.

[5] Liu, Y., Teng, L., Liu, H.-W., Xu, C., Guo, H., Yuan, L., Zhang, X.-B., and Tan, W. (2019) Recent advances in organic-dye-based photoacoustic probes for biosensing and bioimaging, Science China Chemistry 62, 1275-1285. 
[6] Yin, L., Sun, H., Zhang, H., He, L., Qiu, L., Lin, J., Xia, H., Zhang, Y., Ji, S., Shi, H., and Gao, M. (2019) Quantitatively Visualizing Tumor-Related Protease Activity in Vivo Using a Ratiometric Photoacoustic Probe, Journal of the American Chemical Society 141, 32653273.

[7] Wang, Y., Hu, X., Weng, J., Li, J., Fan, Q., Zhang, Y., and Ye, D. (2019) A Photoacoustic Probe for the Imaging of Tumor Apoptosis by Caspase-Mediated Macrocyclization and Self-Assembly, Angewandte Chemie International Edition 58, 4886-4890.

[8] Cheng, P., Chen, W., Li, S., He, S., Miao, Q., and Pu, K. (2020) Fluoro-Photoacoustic Polymeric Renal Reporter for Real-Time Dual Imaging of Acute Kidney Injury, Advanced Materials 32, 1908530.

[9] Wu, Y., Huang, S., Wang, J., Sun, L., Zeng, F., and Wu, S. (2018) Activatable probes for diagnosing and positioning liver injury and metastatic tumors by multispectral optoacoustic tomography, Nature Communications 9, 3983.

[10] Knox, H. J., Hedhli, J., Kim, T. W., Khalili, K., Dobrucki, L. W., and Chan, J. (2017) A bioreducible $\mathrm{N}$-oxide-based probe for photoacoustic imaging of hypoxia, Nature Communications 8, 1794.

[11] Knox, H. J., Kim, T. W., Zhu, Z., and Chan, J. (2018) Photophysical Tuning of N-OxideBased Probes Enables Ratiometric Photoacoustic Imaging of Tumor Hypoxia, ACS Chemical Biology 13, 1838-1843.

[12] Chen, M., Knox, H. J., Tang, Y., Liu, W., Nie, L., Chan, J., and Yao, J. (2019) Simultaneous photoacoustic imaging of intravascular and tissue oxygenation, Opt. Lett. 44, 3773-3776.

[13] Li, H., Zhang, P., Smaga, L. P., Hoffman, R. A., and Chan, J. (2015) Photoacoustic Probes for Ratiometric Imaging of Copper(II), Journal of the American Chemical Society 137, 15628-15631.

[14] Reinhardt, C. J., Zhou, E. Y., Jorgensen, M. D., Partipilo, G., and Chan, J. (2018) A Ratiometric Acoustogenic Probe for in Vivo Imaging of Endogenous Nitric Oxide, Journal of the American Chemical Society 140, 1011-1018.

[15] Reinhardt, C. J., Xu, R., and Chan, J. (2020) Nitric oxide imaging in cancer enabled by steric relaxation of a photoacoustic probe platform, Chemical Science 11, 1587-1592.

[16] Zhang, J., Zhen, X., Upputuri, P. K., Pramanik, M., Chen, P., and Pu, K. (2017) Activatable Photoacoustic Nanoprobes for In Vivo Ratiometric Imaging of Peroxynitrite, Advanced Materials 29, 1604764.

[17] Roberts, S., Seeger, M., Jiang, Y., Mishra, A., Sigmund, F., Stelzl, A., Lauri, A., Symvoulidis, P., Rolbieski, H., Preller, M., Deán-Ben, X. L., Razansky, D., Orschmann, T., Desbordes, S. C., Vetschera, P., Bach, T., Ntziachristos, V., and Westmeyer, G. G. (2018) Calcium Sensor for Photoacoustic Imaging, Journal of the American Chemical Society 140, 27182721.

[18] Zhang, C., Gao, R., Zhang, L., Liu, C., Yang, Z., and Zhao, S. (2020) Design and Synthesis of a Ratiometric Photoacoustic Probe for In Situ Imaging of Zinc lons in Deep Tissue In Vivo, Anal Chem 92, 6382-6390.

[19] Tobkes, A. I., and Nord, H. J. (1995) Liver biopsy: review of methodology and complications, Dig Dis 13, 267-274. 
[20] Oe, S., Honma, Y., Yabuki, K., Morino, K., Kumamoto, K., Hayashi, T., Kusanaga, M., Ogino, N., Minami, S., Shibata, M., Abe, S., and Harada, M. (2020) Importance of a Liver Biopsy in the Management of Wilson Disease, Intern Med 59, 77-81.

[21] Di Tommaso, L., Spadaccini, M., Donadon, M., Personeni, N., Elamin, A., Aghemo, A., and Lleo, A. (2019) Role of liver biopsy in hepatocellular carcinoma, World J Gastroenterol $25,6041-6052$.

[22] Ferenci, P. (2004) Review article: diagnosis and current therapy of Wilson's disease, Aliment Pharmacol Ther 19, 157-165.

[23] Huster, D., Finegold, M. J., Morgan, C. T., Burkhead, J. L., Nixon, R., Vanderwerf, S. M., Gilliam, C. T., and Lutsenko, S. (2006) Consequences of Copper Accumulation in the Livers of the Atp7b-/- (Wilson Disease Gene) Knockout Mice, The American Journal of Pathology 168, 423-434.

[24] Yücel, I., Arpaci, F., Ozet, A., Döner, B., Karayilanoğlu, T., Sayar, A., and Berk, O. (1994) Serum copper and zinc levels and copper/zinc ratio in patients with breast cancer, Biol Trace Elem Res 40, 31-38.

[25] Shanbhag, V. C., Gudekar, N., Jasmer, K., Papageorgiou, C., Singh, K., and Petris, M. J. (2021) Copper metabolism as a unique vulnerability in cancer, Biochimica et Biophysica Acta (BBA) - Molecular Cell Research 1868, 118893.

[26] Adachi, S., Takemoto, K., Ohshima, S., Shimizu, Y., and Takahama, M. (1991) Metal concentrations in lung tissue of subjects suffering from lung cancer, International Archives of Occupational and Environmental Health 63, 193-197.

[27] Zhang, X., and Yang, Q. (2018) Association between serum copper levels and lung cancer risk: A meta-analysis, J Int Med Res 46, 4863-4873.

[28] Hirayama, T., Van de Bittner, G. C., Gray, L. W., Lutsenko, S., and Chang, C. J. (2012) Nearinfrared fluorescent sensor for in vivo copper imaging in a murine Wilson disease model, Proceedings of the National Academy of Sciences 109, 2228.

[29] Heffern, M. C., Park, H. M., Au-Yeung, H. Y., Van de Bittner, G. C., Ackerman, C. M., Stahl, A., and Chang, C. J. (2016) In vivo bioluminescence imaging reveals copper deficiency in a murine model of nonalcoholic fatty liver disease, Proceedings of the National Academy of Sciences, 201613628.

[30] Zhou, E. Y., Knox, H. J., Liu, C., Zhao, W., and Chan, J. (2019) A Conformationally Restricted Aza-BODIPY Platform for Stimulus-Responsive Probes with Enhanced Photoacoustic Properties, Journal of the American Chemical Society 141, 17601-17609.

[31] Wang, S., Sheng, Z., Yang, Z., Hu, D., Long, X., Feng, G., Liu, Y., Yuan, Z., Zhang, J., Zheng, H., and Zhang, X. (2019) Activatable Small-Molecule Photoacoustic Probes that Cross the Blood-Brain Barrier for Visualization of Copper(II) in Mice with Alzheimer's Disease, Angewandte Chemie International Edition 58, 12415-12419.

[32] Festa, R. A., and Thiele, D. J. (2011) Copper: an essential metal in biology, Curr Biol 21, R877-883.

[33] Taki, M., Iyoshi, S., Ojida, A., Hamachi, I., and Yamamoto, Y. (2010) Development of Highly Sensitive Fluorescent Probes for Detection of Intracellular Copper(I) in Living Systems, Journal of the American Chemical Society 132, 5938-5939. 
[34] Lu, S. C. (2020) Dysregulation of glutathione synthesis in liver disease, Liver Research 4, 6473.

[35] Traverso, N., Ricciarelli, R., Nitti, M., Marengo, B., Furfaro, A. L., Pronzato, M. A., Marinari, U. M., and Domenicotti, C. (2013) Role of Glutathione in Cancer Progression and Chemoresistance, Oxidative Medicine and Cellular Longevity 2013, 972913.

[36] Au-Yeung, H. Y., New, E. J., and Chang, C. J. (2012) A selective reaction-based fluorescent probe for detecting cobalt in living cells, Chemical Communications 48, 5268-5270.

[37] Au-Yeung, H. Y., Chan, J., Chantarojsiri, T., and Chang, C. J. (2013) Molecular Imaging of Labile Iron(II) Pools in Living Cells with a Turn-On Fluorescent Probe, Journal of the American Chemical Society 135, 15165-15173.

[38] Alvarez, H. M., Xue, Y., Robinson, C. D., Canalizo-Hernández, M. A., Marvin, R. G., Kelly, R. A., Mondragón, A., Penner-Hahn, J. E., and O'Halloran, T. V. (2010) Tetrathiomolybdate Inhibits Copper Trafficking Proteins Through Metal Cluster Formation, Science 327, 331.

[39] Ludwig, J., Moyer, T. P., and Rakela, J. (1994) The Liver Biopsy Diagnosis of Wilson's Disease: Methods in Pathology, American Journal of Clinical Pathology 102, 443-446.

[40] Khan, G., and Merajver, S. (2009) Copper chelation in cancer therapy using tetrathiomolybdate: an evolving paradigm, Expert Opin Investig Drugs 18, 541-548.

[41] Baldari, S., Di Rocco, G., and Toietta, G. (2020) Current Biomedical Use of Copper Chelation Therapy, Int J Mol Sci 21, 1069.

[42] Chitgupi, U., Nyayapathi, N., Kim, J., Wang, D., Sun, B., Li, C., Carter, K., Huang, W.-C., Kim, C., Xia, J., and Lovell, J. F. (2019) Surfactant-Stripped Micelles for NIR-II Photoacoustic Imaging through $12 \mathrm{~cm}$ of Breast Tissue and Whole Human Breasts, Advanced Materials 31, 1902279.

[43] Liu, Y.-H., Xu, Y., Liao, L.-D., Chan, K. C., and Thakor, N. V. (2018) A Handheld Real-Time Photoacoustic Imaging System for Animal Neurological Disease Models: From Simulation to Realization, Sensors (Basel) 18, 4081.

[44] Taruttis, A., Timmermans, A. C., Wouters, P. C., Kacprowicz, M., Dam, G. M. v., and Ntziachristos, V. (2016) Optoacoustic Imaging of Human Vasculature: Feasibility by Using a Handheld Probe, Radiology 281, 256-263.

[45] Yao, J., and Wang, L. (2014) Photoacoustic brain imaging: from microscopic to macroscopic scales, Neurophotonics 1, 011003.

[46] Tang, J., Coleman, J. E., Dai, X., and Jiang, H. (2016) Wearable 3-D Photoacoustic Tomography for Functional Brain Imaging in Behaving Rats, Scientific Reports 6, 25470.

[47] Yang, J.-M., Chen, R., Favazza, C., Yao, J., Li, C., Hu, Z., Zhou, Q., Shung, K. K., and Wang, L. V. (2012) A 2.5-mm diameter probe for photoacoustic and ultrasonic endoscopy, Opt Express 20, 23944-23953.

[48] Yang, J.-M., Favazza, C., Chen, R., Yao, J., Cai, X., Maslov, K., Zhou, Q., Shung, K. K., and Wang, L. V. (2012) Simultaneous functional photoacoustic and ultrasonic endoscopy of internal organs in vivo, Nature Medicine 18, 1297-1302. 
Figures

a

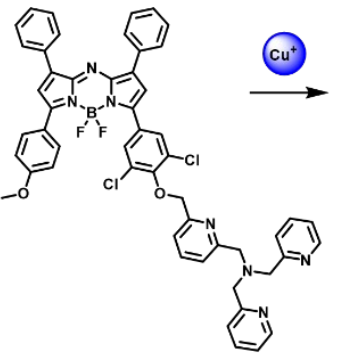

b

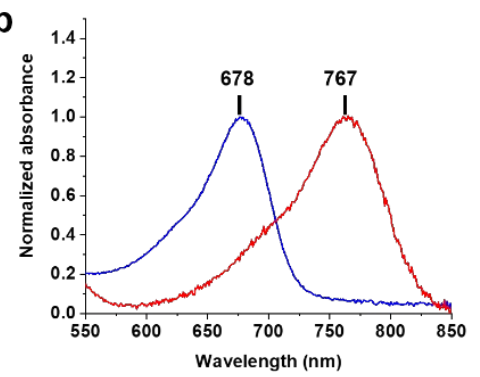

e

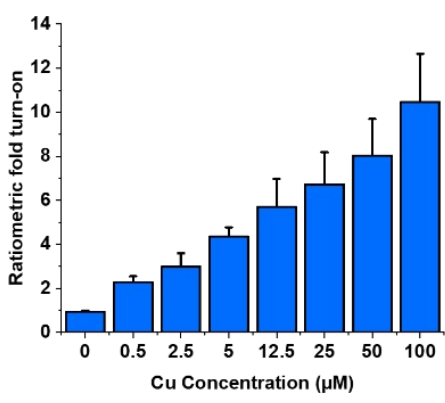

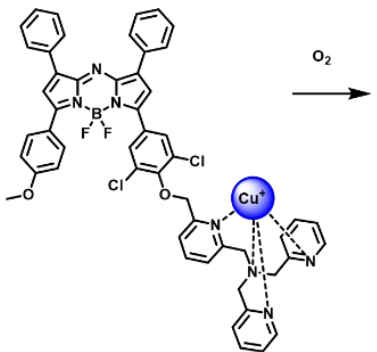
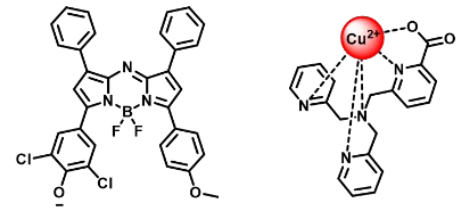

C
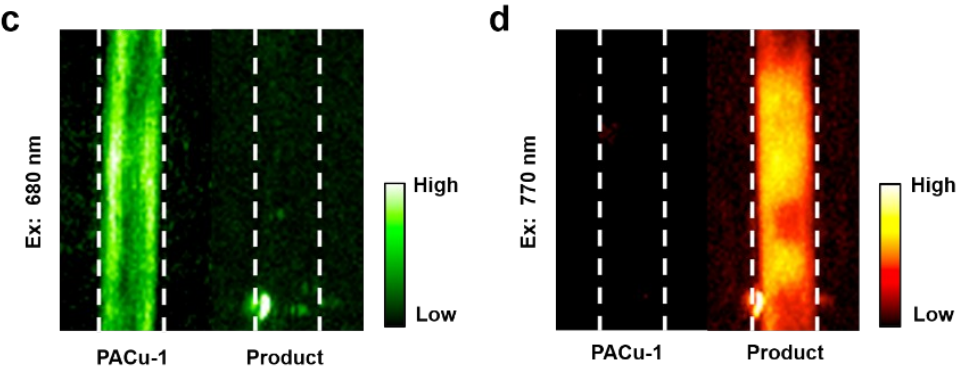

f

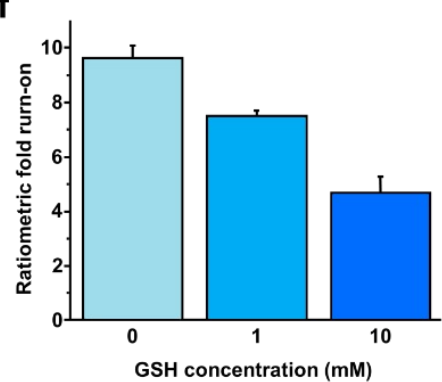

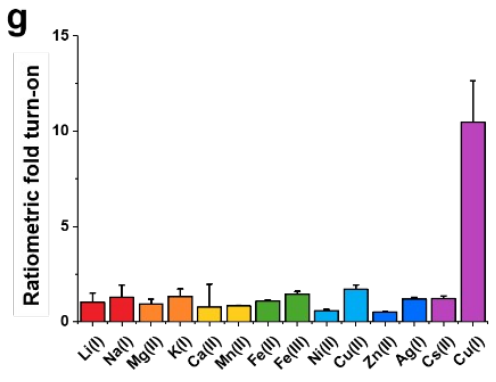

Figure 1. a) General reaction schematic of PACu-1 with $\mathrm{Cu}(\mathrm{I})$. b) Normalized absorbance spectra of PACu-1 (blue) and turnover product (red). PA image of c) PACu-1 and d) the product in a tissue-mimicking phantom excited at 680 and $770 \mathrm{~nm}$. Images compiled from different phantoms e) Ratiometric fold turn-on after incubating PACu-1 with $0,0.5,2.5,5,12.5,25,50$, and $100 \mu \mathrm{M}$ $\mathrm{Cu}(\mathrm{I})$. f) Ratiometric fold turn-on of PACu-1 after incubating with $100 \mu \mathrm{M} \mathrm{Cu}(\mathrm{I})$ and 0,1 , or $10 \mathrm{mM}$ GSH. g) Ratiometric fold turn-on after incubating with $100 \mu \mathrm{M}$ of various metal ions. All assays were performed in 1:1 DMF:HEPES, pH 7.4 for $1 \mathrm{~h}$. Values are reported as mean $\pm \operatorname{SD}(n=3)$. 

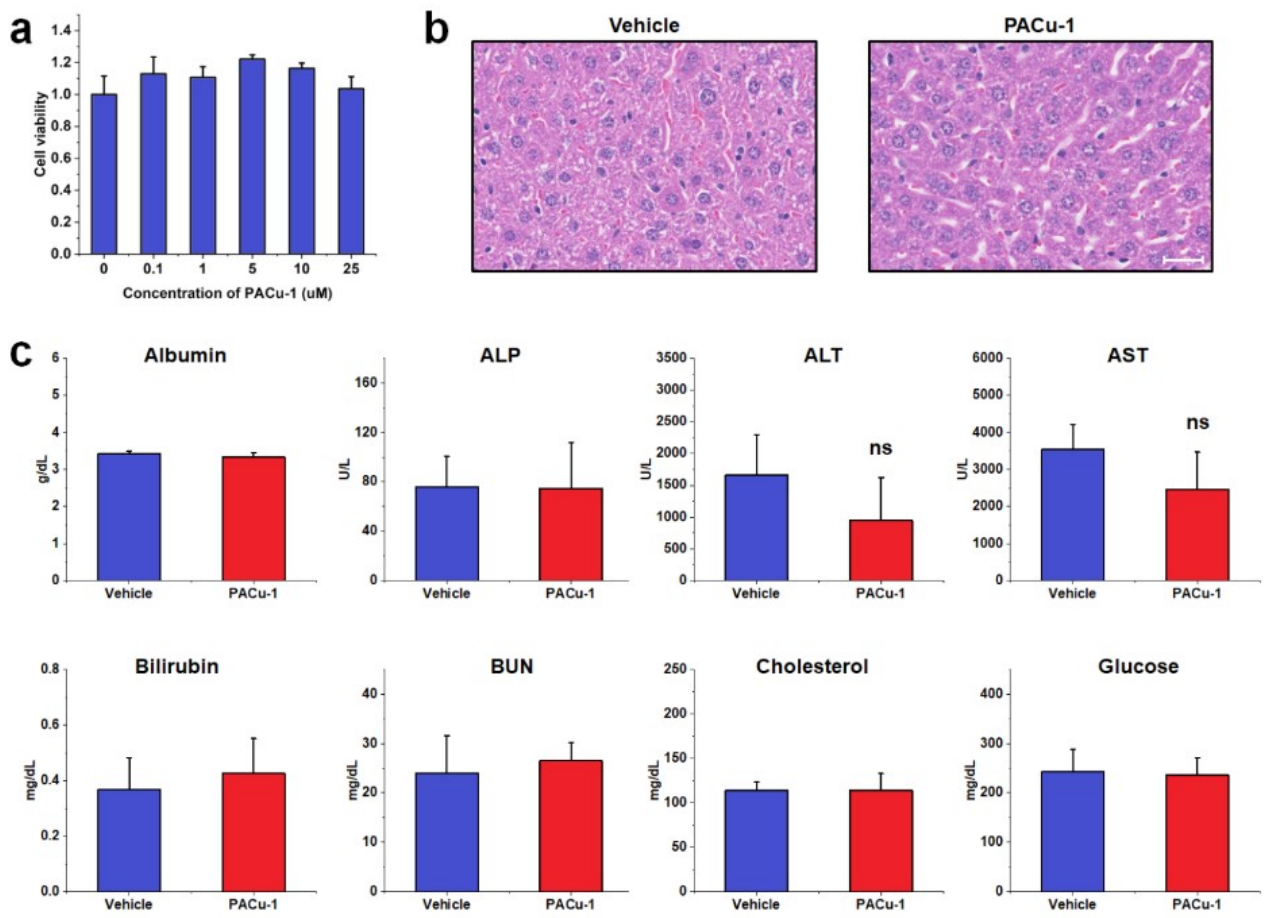

Figure 2. a) MTT assay measuring cell viability after incubating cells with $0,0.1,1,5,10$, and 25 $\mu \mathrm{M}$ of PACu-1 for $24 \mathrm{~h}$. b) H\&E stains of the liver after treating live mice with 0 or $50 \mu \mathrm{M}$ of PACu1. c) Liver function assays on serum after treating live mice with 0 or $50 \mu \mathrm{M}$ of PACu-1. Values are reported as mean \pm SD $(n=10)$. 
a
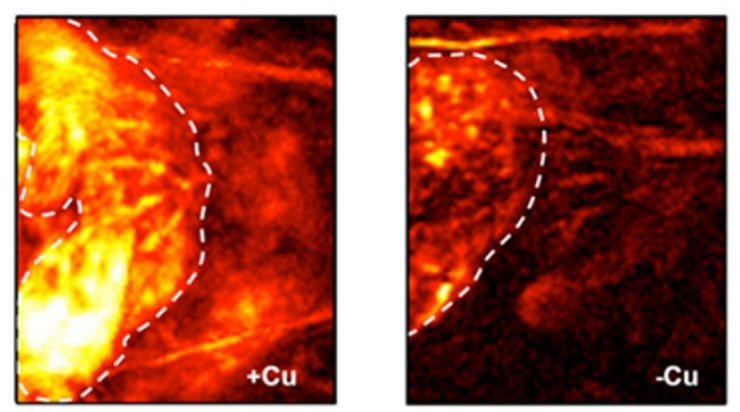

b
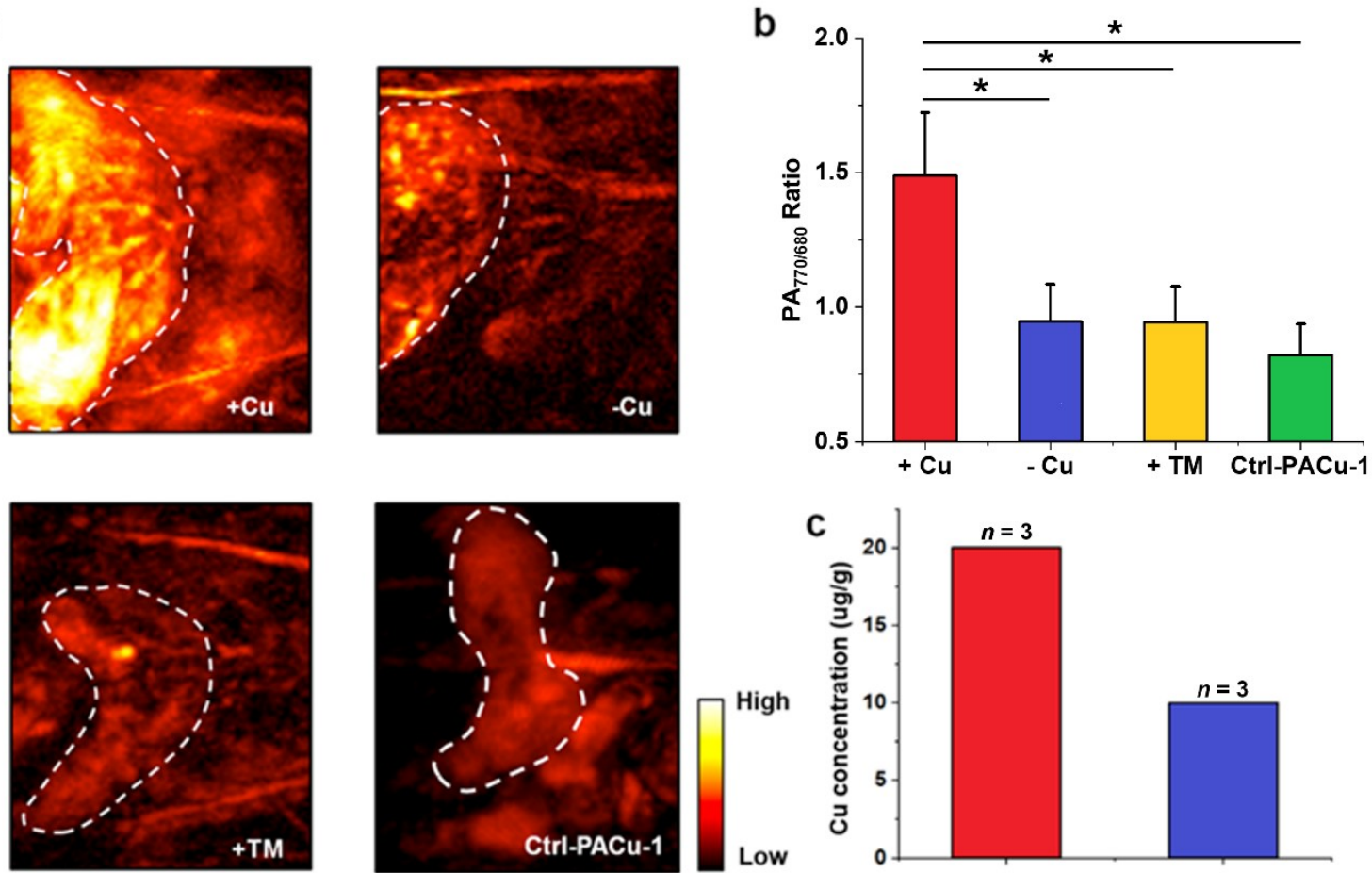
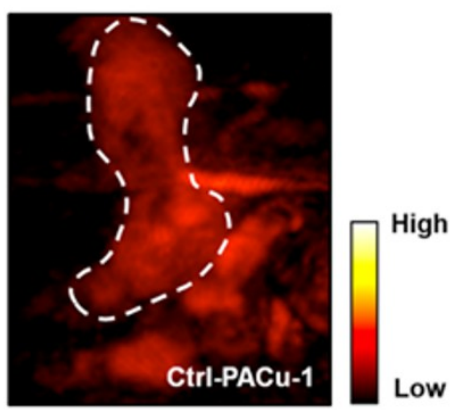

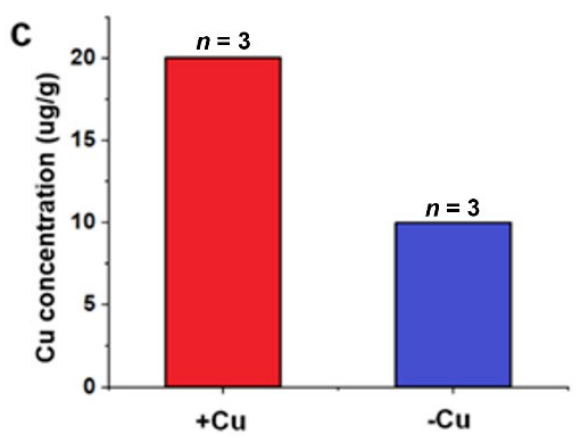

Figure 3. a) Representative PA images of the liver (dashed white line) using PACu-1 (50 $\mu \mathrm{M})$ and pre-treatment with $0 \mathrm{mg} / \mathrm{kg} \mathrm{CuCl}_{2}(n=5)$ or $5 \mathrm{mg} / \mathrm{kg} \mathrm{CuCl}_{2}(n=4)$, ammonium tetrathiomolybdate $(n=5)$, or Ctrl-PACu-1 (50 $\mu \mathrm{M})(n=4)$. b) PA ratiometric fold turn-on for matching conditions in a. c) $\mathrm{Cu}$ concentration in the liver after pretreatment with $0 \mathrm{mg} / \mathrm{kg} \mathrm{CuCl} 2$ or $5 \mathrm{mg} / \mathrm{kg} \mathrm{CuCl}$ obtained via ICP-MS. Liver ROIs are for visualization purposes and was not used for quantification. Values are reported as mean \pm SD. Statistical analyses were performed using the Kruskal-Wallis Test; * $\mathrm{p}<0.05$. 

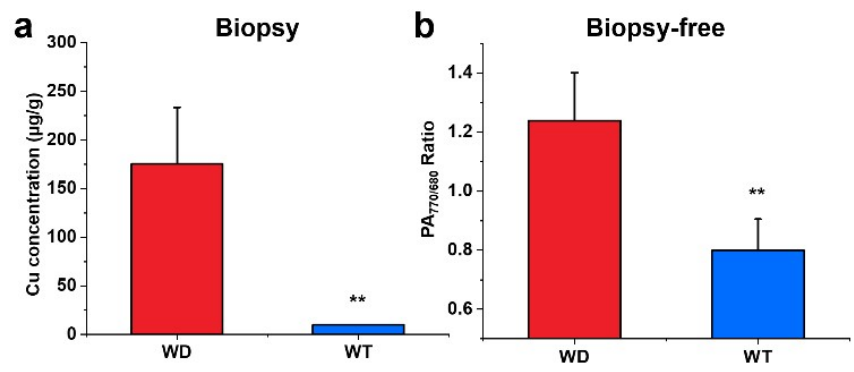

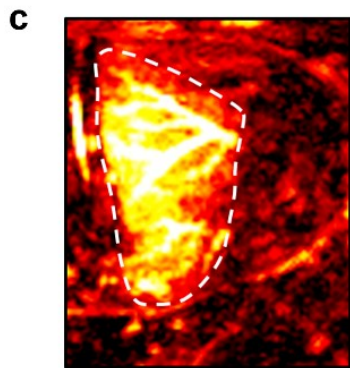

WD

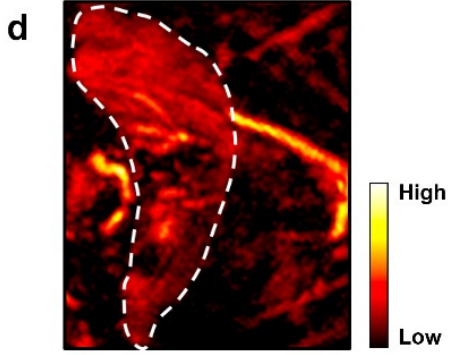

WT

Figure 4. a) Cu concentration in the liver of WD and WT mice $(n=4)$ obtained via ICP-MS. b) PA ratiometric fold turn-on after treatment with PACu-1 (50 $\mu \mathrm{M}, 10 \%$ DMSO in saline) in live WD ( $\mathrm{n}=$ 7) and WT $(n=3)$ mice. c) Representative PA image of the liver after treatment with PACu-1 (50 $\mu \mathrm{M}, 10 \%$ DMSO in saline) in live WD or d) WT mice. Liver ROIs are for visualization purposes and was not used for quantification. Values are reported as mean \pm SD. Statistical analyses were performed using the Student's t-test; ${ }^{\star \star} p<0.01$. 
a

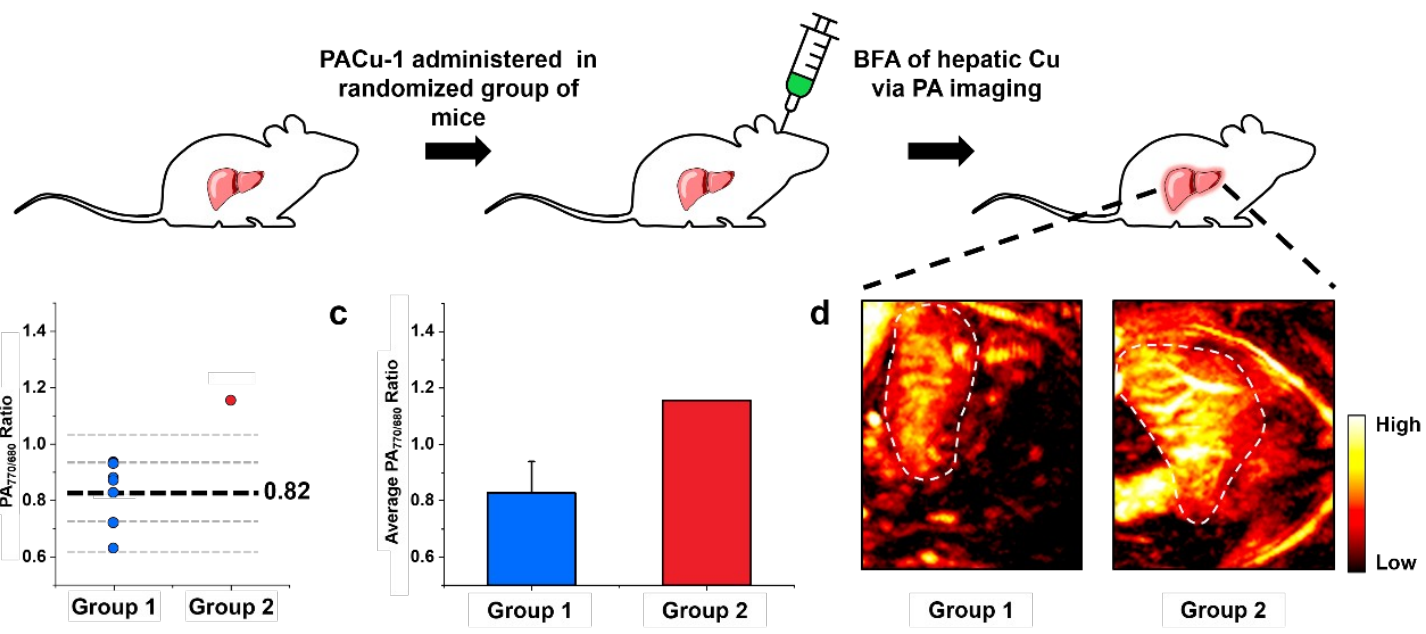

Figure 5. a) General schematic depicting experimental setup of the blind imaging study. b) PA ratiometric fold-turn-on using PACu-1 stratified into groups 1 and 2 based on diagnostic threshold. Total mice in the blind study were $8(W T=7, W D=1)$. c) Average PA ratiometric fold turn-on shown in b. d) Representative PA images of livers (white dashed line) in groups 1 and 2. Liver ROIs are for visualization purposes and was not used for quantification. Values are reported as mean $\pm \mathrm{SD}$. 

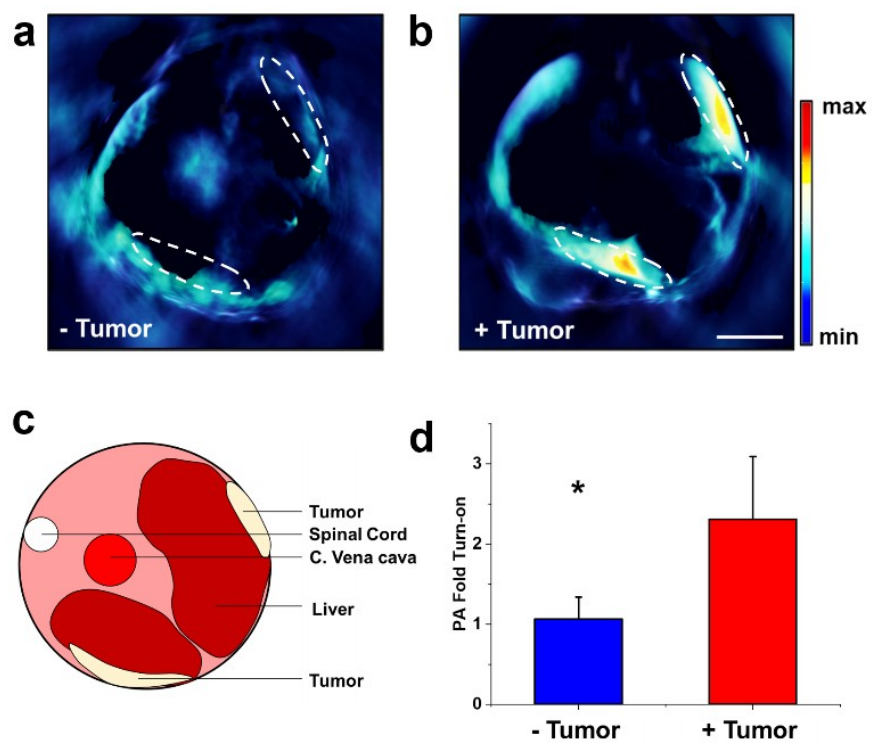

Figure 6. a) Cross section schematic of a mouse to reference the positioning of the liver and tumor. Representative spectrally unmixed MSOT images of the liver $1 \mathrm{~h}$ post-injection in $\mathrm{b}$ ) nontumor bearing mice and c) tumor bearing mice. Scale bar represents $5 \mathrm{~mm}$. d) Average PA signal from MSOT imaging represented in Figure $6 \mathrm{~b}(n=3)$ and Figure $6 \mathrm{c}(n=5)$. Statistical analysis was performed using the Student's t-test, *: $p<0.05$. 